\title{
L’usage Du Wolof En Milieu Urbain : Évolution Vers De Nouvelles Règles Ou Perte D'authenticité?
}

\author{
Sokhna Bao Diop, \\ Université Gaston Berger, Saint-Louis, Sénégal
}

Doi:10.19044/esj.2019.v15n35p211 URL:http://dx.doi.org/10.19044/esj.2019.v15n35p211

\section{Résumé}

A l'instar de beaucoup de pays d'Afrique, le Sénégal se distingue par une diversité linguistique se caractérisant par la présence de nombreuses langues nationales ${ }^{18}$ qui coexistent avec des langues étrangères comme le français, l'anglais, le portugais et l'arabe. Ce contact linguistique a fait que les langues nationales subissent l'influence des langues étrangères. Ainsi le wolof, langue véhiculaire du pays, est au centre de ce rendez-vous du donner et du recevoir. En effet, cela a favorisé chez les locuteurs wolof, particulièrement ceux des milieux urbains, la présence de phénomènes tels que l'emprunt, le code-switching, le code-mixing, etc. Cette situation n'est pas sans conséquences dans la mesure où, comme l'a affirmé Moussa DAFF ${ }^{19}$, la majeure partie des locuteurs wolof ont du mal à soutenir une conversation en langue wolof sans commettre des écarts ou bien sans employer des termes empruntés aux autres langues. Cet article vise à démontrer, dans une démarche empirique, combien cette influence a eu un impact sur l'usage du wolof de nos jours en milieu urbain. Avec la méthode d'observation participante, les données recueillies par enregistrement sont analysées selon les cadres théoriques de la linguistique descriptiviste fonctionnaliste. Les résultats révèlent que l'impact ne s'arrête pas aux emprunts ou au mélange de codes observés dans le "wolof urbain ancien », mais va au-delà et conduit à l'existence d'un « wolof urbain contemporain » comportant non seulement les interférences avec les langues étrangères mais aussi et surtout le non-respect des règles d'usage de la langue témoignant de l'écart considérable par rapport au parler « authentique ».

Mots-Clés : Langues Nationales, Contact De Langues, Influence, Mutation, Wolof Urbain

\footnotetext{
${ }^{18}$ Une trentaine, selon les recensements de l'Agence Nationale de la Statistique et de la Démographie (ANSD)

${ }^{19}$ Moussa DAFF (1998 : http://www.uquebec.ca/diverscite)
} 


\title{
The Use of Wolof in Urban Areas: Evolution Towards New Rules or Loss of Authenticity?
}

\author{
Sokhna Bao Diop, \\ Université Gaston Berger, Saint-Louis, Sénégal
}

\begin{abstract}
Like many African countries, Senegal is distinguished by a linguistic diversity characterized by the presence of many national languages that coexist with foreign languages such as French, English, Portuguese and Arabic. This linguistic contact has meant that the national languages are influenced by foreign languages. Thus Wolof, the lingua franca of the country, is at the center of this meeting of giving and receiving. In fact, this has favored in Wolof speakers, particularly those from urban areas, the presence of phenomena such as linguistic borrowing, code-switching, code-mixing and so on. This situation is not without consequences since, as stated by Moussa DAFF, the majority of Wolof speakers find it difficult to sustain a conversation in Wolof without committing language differences or using terms borrowed from other languages. This article aims at demonstrating, in an empirical approach, how much this influence has had an impact on the use of Wolof today in urban areas. With the participatory observation method, the data collected by recording are analyzed according to the theoretical frameworks of functionalist descriptivist linguistics. The results reveal that the impact does not stop with borrowing or the mixture of codes observed in the "old urban Wolof", but goes beyond and leads to the existence of a "contemporary urban Wolof" comprising not only the interferences with foreign languages but also and above all the non-respect of the rules of use of the language testifying to the considerable difference compared to the "authentic" speech.
\end{abstract}

Keywords: National Languages, Language Contact, Influence, Mutation, Urban Wolof

\section{Introduction}

La planète est comparée à une tour de Babel où vivent ensemble diverses communautés linguistiques. Ainsi, il a été répertorié «à la surface du globe entre 6500 et 7000 langues » (Calvet, 2004, p. 287). Les déplacements des populations ont favorisé le contact de cultures et de langues. Le continent africain en général, et le Sénégal en particulier, qui est un espace où règne le 
multilinguisme, n'échappe pas à cette situation de contact de langues. Ce dernier a, depuis longtemps, suscité un intérêt notoire dans les études en Linguistique et Sociolinguistique. Il existe de nombreux travaux portant sur le contact entre les langues et les phénomènes qui en découlent. Au Sénégal, la plupart de ces travaux s'intéressent au contact entre le wolof (langue véhiculaire) et le français (langue officielle). Ils se focalisent particulièrement sur les phénomènes d'emprunt linguistique (Dumont, 1983 \& Ngom, 2005), de code-mixing et de code-switching (Thiam, 1994 ; Daff, 1998 \& Kebe, 2011) principalement observés en milieux urbains. Ces études, aussi pertinentes qu'intéressantes, se limitent à la description de ces phénomènes, mais ne rendent pas compte de l'ensemble des faits attestés dans les pratiques discursives des locuteurs wolof. De ce fait, un travail prenant en charge, dans une démarche empirique, l'analyse descriptive des changements inhérents à la grammaire de la langue reste à faire. En effet, outre les phénomènes cités cidessus, l'on observe dans le «wolof urbain contemporain » des écarts au regard des normes d'usage de la langue wolof. L'objectif de cette contribution est de décrire les changements observés dans l'usage du wolof en milieu urbain, aussi bien sur le plan phonologique que morphosyntaxique. Après une présentation sociolinguistique de la langue wolof et la discussion sur l'origine et la définition du phénomène «wolof urbain », l'étude aborde la description et l'analyse des phénomènes linguistiques observés dans cette façon de parler ; puis se focaliser sur les causes et conséquences de tels phénomènes avant de donner des perspectives.

\section{Le cadre de l'étude}

\subsection{Le contexte}

Cette étude est menée dans un contexte multilingue où, comme cela a déjà été souligné, le wolof $\mathrm{a}$, depuis des siècles, été en contact avec d'autres langues. L'on peut noter sa cohabitation avec, d'une part, les langues autochtones et d'autre part, les langues étrangères. Selon Pathé Diagne (1971 : 11) : «Le monde culturel défini par le wolof déborde le cadre d'une ethnie. La langue a pris racine à partir de la région du Lôf, ancienne province du Tekrour, puis du Wâlo.[...] Elle a [...] reçu des apports divers du côté du Pular et du Serer dont on la rapproche traditionnellement. Par rapport au Mandingue non plus l'influence n'a pas été négligeable ».

Ceci montre que certaines langues autochtones du Sénégal sont génétiquement apparentées et cela facilite bien évidemment les apports linguistiques réciproques qui se produisent entre elles.

Cependant, il semble que l'impact des autres langues autochtones sur le wolof n'est pas aussi important que celui des langues étrangères. En effet, comme le fait remarquer Diagne : «La présence islamique très ancienne y a laissé des traces profondes. Les réalités véhiculées par l'essor des cultures 
urbaines et techniciennes n'ont pas manqué de susciter un renouvellement profond de son lexique et d'y déposer quantités de vocables portugais, anglais et français ${ }^{20} \gg$.

Les langues étrangères ont eu et continuent d'avoir une très forte influence sur le wolof. Ceci est à l'origine du grand degré de métissage et des changements qui s'observent dans l'usage de la langue wolof par les locuteurs natifs et principalement ceux des milieux urbains. Dans une approche synchronique, cette étude s'intéresse à l'état de l'usage de la langue wolof de nos jours, en milieu urbain.

\subsection{La méthodologie}

La collecte des données et l'analyse linguistique se sont appuyées sur les cadres théoriques de la linguistique descriptiviste fonctionnaliste. Cette méthode permet de considérer les usages qui sont faits de la langue wolof à un moment donné, d'où l'approche synchronique de l'étude.

Le corpus recueilli est principalement constitué de phrases transcrites, annotées et traduites. Pour l'obtenir, on a exploité les liens d'appartenance à la communauté wolof en utilisant la méthode d'observation participante qui « consiste à recueillir des données en participant soi-même aux situations qui les produisent » (Blanchet, 2012, p. 46). Il s'agit, en effet, de conversations spontanées auxquelles on a participé ou bien assisté dans la vie quotidienne. Le corpus a été recueilli à Saint-Louis, Louga et Dakar auprès de locuteurs natifs. Le choix de ces villes s'explique par le fait qu'on s'y rend assez souvent à causes des attaches familiales ou bien dans le cadre d'activités professionnelles. En outre, elles répondent aux critères d'enquête qu'on s'est fixés notamment l'urbanisation, entre autres.

Avec un minimum de contrôle, les données recueillies sont d'un grand degré de spontanéité de la part des locuteurs enregistrés. En effet, elles ont été recueillies dans des situations diverses (des conversations entre adultes, des conversations entres jeunes, des négociations au marché, des dialogues entre parents et enfants, etc.), qu'on n'a pas créées et qui auraient eu lieu sans nous. L'objectif principal était de saisir leur langage en « toute liberté » et d'en identifier les types d'interactions ponctuelles présentant les problèmes visés. On ne s'est pas limité aux enregistrements. La prise de notes nous a également été d'un grand apport.

Pour ce qui concerne la transcription du corpus, on a choisi l'orthographe officielle de la langue sans indications de pauses, rires, hésitations, rythmes, etc. pour se simplifier la tâche, mais tout en veillant à ne pas ajouter ou enlever un élément de la production. Dans la présentation, on a 
fait le choix de proposer une ligne de transcription orthographique, puis une ligne pour la glose et enfin une ligne pour la traduction en français.

\section{La langue wolof}

\subsection{Présentation}

Le terme wolof désigne à la fois la langue et l'ethnie (les gens qui parlent la langue). Le wolof est classé dans la branche nord des langues ouestatlantiques de la famille Niger-Congo. Cette branche à laquelle appartient le wolof se subdivise en 4 sous-groupes que sont : Sénégalo-Gambien (wolof, seereer sine, pulaar), Cangin (noon, ndut, saafeen, paloor), Bak (joolaa) et Tenda (bédik, biafada). C'est une langue transfrontalière, essentiellement parlée dans trois pays que sont le Sénégal, la Gambie et la Mauritanie. Au Sénégal on estime le nombre de locuteurs du wolof à plus de $85 \%$ de la population même si près de $42 \%$ seulement sont des locuteurs natifs. Les foyers traditionnels du wolof sont le Djolof, le Cayor, le Baol, le walo, le Saloum. C'est une langue relativement homogène qui présente néanmoins une variante : le lébu parlé essentiellement sur la petite côte.

La constitution du Sénégal stipule dans son article premier que « la langue officielle de la République du Sénégal est le français. Les langues nationales sont le Diola, le Malinké, le Pular, le Sérère, le Soninké, le Wolof et toute autre langue nationale qui sera codifiée ». Le wolof, ayant été codifié depuis 1971, c'est-à-dire dotée d'un système de transcription orthographique et de règles de séparation des mots, reste la principale langue véhiculaire du Sénégal, car permettant à des communautés de langues différentes de communiquer. Il est généralement utilisé dans les transactions commerciales, dans les médias, dans le système éducatif et à l'assemblée nationale. Les autres langues ont le statut de langues vernaculaires. En d'autres termes, langues qui ne sont utilisées qu'à l'intérieur d'une communauté donnée.

Par rapport à ses caractéristiques typologiques, le wolof est de structure syntaxique SVO (sujet, verbe, objet). C'est une langue agglutinante qui n'a pas de ton et fonctionne avec des classes nominales.

\subsection{Le phénomène «wolof urbain » : origine et définition}

Un parler urbain est une forme de la langue utilisée par les populations qui habitent la ville. C'est donc un type de parler directement rattaché à la ville et qui a des règles qui lui sont particulières du fait que ce lieu soit un melting pot où on rencontre différentes communautés linguistiques. Pour ce qui concerne le wolof, le phénomène est, pour beaucoup d'auteurs, le résultat du contact entre le wolof et le français. Ainsi les formes d'usage du wolof où l'on trouve des mots français sont assimilées à ce que ces auteurs nomment «wolof urbain ».

Ce dernier (qui provient donc du contact entre le wolof et le français 
selon la conception de certains auteurs) ne daterait pas de la période d'après les indépendances. Cette opinion est défendue par la linguiste Fiona McLaughlin (2008, p. 714) de l'université de Floride. Selon elle, "urban Wolof, $[\ldots]$, is in fact a variety with a long history, dating back certainly to the mid-19th century, and possibly as far back as the 18th" (le Wolof urbain, [...], est en fait une variété avec une longue histoire qui remonte certainement au milieu du XIXe siècle et peut-être même jusqu'au XVIIIe siècle). Cette affirmation peut se comprendre dans la mesure où les wolof ont été les premiers en contact avec l'administration coloniale française et les premiers formés par celle-ci. En effet, en milieu wolof, virent le jour les premiers comptoirs commerciaux, les premières écoles françaises ainsi que l' installation du chemin de fer entre Saint-Louis et Dakar qui, respectivement, furent capitales de l' $\mathrm{AOF}^{21}$. Selon l'auteur, le phénomène « wolof urbain » serait né à Saint-Louis (où eurent lieu les premiers contacts entre le wolof et le français) vers la fin du XIXe siècle. Pour confirmer son hypothèse, elle cite deux documents historiques datant de 1864 : l'un fut rédigé par le gouverneur Faidherbe qui répertoria 1.500 mots français et leurs correspondants en wolof, pulaar et soninké. L'autre document est un recueil de 1.200 phrases usuelles avec leur traduction en wolof qui fut élaboré par Louis Descemet qui essaya de décrire la façon dont parlait la société métisse saint-louisienne de l'époque.

A la lumière de ces propos, on constate que le concept « wolof urbain » est confiné au mélange entre le wolof et le français. Si le «parler urbain » provient du contact de langues, cette conception semble très restreinte dans la mesure où le français n'est pas la seule langue avec laquelle le wolof a été en contact. Bien avant la colonisation, le wolof a été en contact avec l'arabe lors $\mathrm{du}$ processus d'islamisation. Le wolof a également beaucoup emprunté à l'arabe, notamment des concepts liés au temps (comme les jours de la semaine), mais aussi aux pratiques religieuses de l'islam. On peut aussi dire la même chose à propos du portugais et de l'anglais dans une moindre mesure. Un grand nombre de vocables provenant de ces langues est présent dans le lexique wolof. Ainsi peut-on se permettre d'avancer que les interférences ne se limitent pas seulement au français, mais englobent aussi les autres langues. Ceci amènerait à avoir une vision beaucoup plus large du phénomène qui ne renfermerait pas seulement deux composantes, mais plusieurs qui donnent au « parler urbain » son caractère hybride. Cela permet de ne pas restreindre le sens du concept au contact entre le wolof et le français. Cependant, les interférences ne sont pas, non plus, les seuls changements que l'on peut noter dans le «wolof urbain », particulièrement celui de nos jours. Nous observons, en effet, des changements d'ordre phonologique et morphosyntaxique permettant de faire la distinction entre le « wolof urbain » que décrit cet article

\footnotetext{
${ }^{21}$ Afrique Occidentale Française
} 
et celui décrit par Fiona McLaughlin. On est donc en présence de deux types de « wolof urbain » : un ancien comportant uniquement les interférences avec les langues étrangères et un autre contemporain comportant à la fois les interférences avec les langues étrangères mais aussi le non-respect des règles d'usage de la langue. Ce dernier, qu'on nommerait le «wolof urbain contemporain » en relation avec le contexte socio-culturel actuel paraît beaucoup plus complexe et mérite d'être un objet d'étude pour dévoiler ses principales caractéristiques.

\section{Description et analyse des phénomènes attestés dans l'usage du wolof en milieux urbains}

Les mutations observées dans l'usage du «wolof urbain contemporain » sont principalement de deux catégories : les phénomènes d'ordre sociolinguistique résultant du contact de langues et les phénomènes d'ordre linguistique résultant du non-respect des règles grammaticales. La première aborde les phénomènes d'interférences linguistiques (l'emprunt, le code-switching et le code-mixing) tandis que la seconde touche les aspects phonologique et morphosyntaxique de la langue. Il faut noter que la question du contact du wolof avec d'autres langues ayant fait couler beaucoup d'encre, l'analyse abordera très rapidement ce point avant de mettre l'accent sur les phénomènes relatifs au non-respect des normes linguistiques telles que définies dans la grammaire du wolof.

\subsection{Les phénomènes d'ordre sociolinguistique :}

Ils tournent autour de l'emprunt, du code-switching, du code-mixing, entre autres.

De nos jours, on trouve de plus en plus dans le discours des locuteurs wolof des mots provenant des langues étrangères comme le français, l'anglais et l'arabe. Cependant si on devait proposer un classement du degré d'interférence de ces langues, le français l'emporterait largement. En effet, le corpus recueilli témoigne de cela du fait qu'il ne comporte que des éléments provenant du contact entre le français et le wolof. Cela est peut-être dû au fait qu'il soit la langue officielle du Sénégal : utilisée dans l'administration et l'éducation.

\subsubsection{L'emprunt}

Les chercheurs en distinguent deux catégories, mais chacun y va de sa propre conception du phénomène pour trouver une dénomination. Ndiassé Thiam (1994, p. 18) les désigne sous les noms d'«emprunts intégrés ou établis » et « emprunts spontanés ». Selon lui, ils renvoient respectivement aux termes qui n'existent pas dans le vocabulaire wolof et qui ne subissent pas de changement phonologique et ceux qui sont bien intégrés dans le système phonologique wolof. Kébé (2011, p. 25), pour sa part, utilise les appellations «emprunts consolidés » et «emprunts établis » et fait ainsi, respectivement, 
référence aux éléments du lexique français qui ne subissent pas de changement phonologique une fois utilisés dans un discours en wolof et ceux qui en subissent. On constate chez les deux une différence dans la conception de ce qui est « établi ». Dans cette étude, à la lumière des exemples tirés du corpus, on préfère parler d'emprunts «nécessaires » et d'emprunts de «luxe». Ce choix est guidé par le constat fait de l'usage du wolof de nos jours. En effet, les locuteurs ont, le plus souvent, tendance à employer des mots français sans se soucier du fait que ceux-ci puissent exister dans leur langue. Dans les exemples 1 et 2 (société et agressions) on a des cas d'emprunts que l'on peut considérer comme «nécessaires ». Ils n'ont pas d'équivalents en langue wolof, néanmoins avec des efforts il est possible de jouer avec les mots pour trouver dans le lexique wolof des termes qui se rapprocheraient de leur sens. Cependant, dans 3 et 4 (pàppaam, divorcer et langues) nous assistons à des cas d'emprunts de «luxe » étant donné que ces mots ont bel et bien leurs équivalents en langue wolof. Ceci peut être dû soit à l'ignorance du vocabulaire de la langue soit à un complexe d'infériorité.

1. Seen société bi moo jaxasoo

Leur-société-la-elle-être.en.désordre

Leur société est en désordre

2. Dëkk bi agressions yi bari na

Pays-le-agressions-les-nombreuses-3sg.conj.perfec.

Il y a beaucoup d'agressions dans le pays

3. Pàppaam ak yaayam dañoo divorcer

Père.3sg.poss-et-mère-ils-divorcer

Son père et sa mère ont divorcé

4. Dëkk ba mu nekk langues yu bari lañu fay wax

Pays-où-il-être-langues-qui-nombreuses-on-y.acc-parler

Il parle plusieurs langues dans le pays où il est

3.1.2. Le code-switching

Il y a code-switching lorsque le locuteur d'une langue utilise, en alternance, plus d'une langue au cours d'une même conversation, d'un même discours. Elle peut être intra-phrastique ou inter-phrastique.

Ce phénomène est également observé dans le « wolof urbain contemporain », particulièrement chez les locuteurs instruits. Généralement, on observe dans leurs discours l'alternance entre le wolof et le français. Comme symbolisé dans 5 et 6 , ils commencent leurs propos dans une langue et terminent par l'autre. Ce qui traduit leur incapacité à soutenir un discours purement wolof.

5. Bëgg naa ko laaj quelles sont les raisons

Vouloir-1sg.conj.perfec-lui-demander-quelles-sont-les-raisons

Je veux lui demander les raisons 
6. Mi ngi jokkoo ak ñoom kay d'après eux sa grande sœur avait fait pareil

Elle-prés-être.en.contact-avec-eux-part.insist-d'après-eux-sa-grandesœur-avait-fait-pareil

Elle communique avec eux, apparemment sa grande sœur avait fait pareil

\subsubsection{Le code-mixing}

Comme l'emprunt, le code-mixing est aussi très fréquent. En effet, les locuteurs utilisent dans leurs discours le wolof et les langues étrangères de manière concomitante. Il s'agit d'un discours mixte dans lequel on note la présence de beaucoup d'éléments provenant de deux codes. Les exemples suivants en sont des illustrations parfaites.

7. $\mathrm{Bu} \tilde{n} u$ amoon ci seen variété de fruits ak légumes yi kon mu baax

Si-nous-avoir.pass-prép.loc-vos-variétés-de-fruits-et-légumes-lesalors-il-bon

Ce serait bien si on pouvait avoir vos variétés de fruits et légumes

8. Man amuma aucun intérêt $c i$ sosal qui que ce soit

moi-avoir.1sg.conj.nég-aucun-intérêt-prép.loc-diffamer-qui-que-cesoit

Je n'ai aucun intérêt à diffamer qui que ce soit

9. Est-ce que nit boobu am na un peu de pitié ci jabaram

est-ce-que-personne-cette-avoir-3sg.conj.perfec-pitié-enversfemme.3sg.poss

Est-ce cette personne éprouve de la compassion pour sa femme

L'on observe aussi des interférences faisant intervenir dans un même mot des éléments provenant de deux langues (wolof et langue étrangère). En citant Lafage, Ngom (2005, p.155) les appelle « hybridations lexicales ». Les éléments wolof de ces ensembles sont généralement des morphèmes (verbaux ou nominaux) qui viennent se rattacher à leur contrepartie étrangère (radicaux verbaux, nominaux, adjectivaux). Ils sont illustrés par les exemples cidessous :

10. Attaayaam bi tay saful suukar

Attaaya.3sg.poss-le-aujourd'hui-relevé.nég-sucre

Aujourd'hui son thé n'est pas relevé en sucre

11. Suba nga envoyeel ma wari meer Faatumata

Demain-tu-envoyer.imp-moi-wari-mère-Fatoumata

Tu enverras un wari à mère Fatoumata pour moi demain

12. Am na soxna yu cotiséegul

Avoir-3sg.conj.perfec-femmes-qui-côtiser.accomp.nég

Il y a deux femmes qui n'ont pas encore cotisé 


\section{Li nga wax booy bi naayisul dara quoi}

Ce.que-tu-dire-fille-la-joli.nég-du.tout-quoi

Ce n'est pas du tout bien ce que tu as dit au garçon

Outre ces phénomènes catégorisés d'ordre sociolinguistique parce qu'étant en relation avec le milieu social, on observe d'autres, purement linguistiques.

\subsection{Les phénomènes d'ordre linguistique :}

La langue est un ensemble de signes et un ensemble de règles. Ces dernières président à la formation des mots, des groupes et d'unités plus larges. La mise en fonctionnement de toute langue nécessite l'application plus ou moins rigoureuse de ces règles. Le wolof n'échappe pas à cet état de fait. Cependant il a été observé des phénomènes d'ordre linguistique englobant deux catégories : les changements de type phonologique et les changements de type morphosyntaxique.

\subsubsection{Les changements phonologiques}

Il convient de préciser qu'il ne s'agit pas de variantes régionales en rapport avec les anciens foyers d'implantation de la langue wolof (Kayoor, Bawol, Njàmbur, Jolof, Waalo), mais de véritables changements dont les causes seront discutées dans le point quatre de l'étude.

Certains phonèmes de la langue subissent des changements dans la manière d'articuler. C'est le cas de la consonne prénasale /nd/ qui devient, dans certains mots, simplifiée en une consonne nasale $/ \mathrm{n} /$. Ces phonèmes sont tous des alvéolaires, mais le mode d'articulation qui les distingue fait que la nasale $/ \mathrm{n} /$ est beaucoup plus simple à articuler que la prénasale $/ \mathrm{nd} /$. C'est probablement la raison pour laquelle on trouve dans 14 et 15 nax pour $\boldsymbol{n d a x}$ (parce que) et nànk pour ndànk (doucement).

14. Nax fii nii...

Parce que-ici-comme ça...

Parce qu'ici...

\section{Defal nànk balaa muy tuuru}

Faire.imp-doucement-avant-il.inacc-se.renverser

Fait doucement avant que ça se renverse

Par ailleurs, la consonne palatale /c/ de la préposition locative $\mathrm{ci}$ (dans, à, sur, $y, a u$, etc.) est de nos jours très souvent remplacée par la consonne alvéolaire /s/. En effet, comme l'illustrent les exemples 16 à 18, il semble même que la forme en $s i$ est plus à portée de main des locuteurs de sorte qu'ils oublient la structure originelle de cette préposition. 
16. Ni nekk si ginnaaw...

Ceux-être-au-derrière

Ceux qui sont derrière

17. Loolu dan si sonn

Cela-nous-y-fatigués

Nous en sommes fatigués

18. Li ñu bëgg mooy ñëpp jàpp si

Ce que-nous-vouloir-être-tous-prendre-y

Nous voulons le soutien de tout un chacun

Les consonnes uvulaires simple /q/ et prénasale /nq/ ont également tendance à être respectivement remplacées par les vélaires $/ \mathrm{k} /$ et $/ \mathrm{nk} /$. Beaucoup de locuteurs, spécialement les jeunes, ne font plus l'effort de prononcer ces consonnes comme il se doit. Cela peut se justifier par le fait que le lieu d'articulation de ces uvulaires se situe au niveau de la luette rendant ainsi leur production beaucoup plus complexe que celle des vélaires. Dans les exemples 19 et 20, les mots dàq «chasser » et tanq «puiser» deviennent respectivement dàkk et tank.

19. Dàkkal weñ yi te muur mburu mi

Chasser.imp-mouche-les-et-couvrir.pain.le

Chasse les mouches et couvre le pain

20. Man nga sanguji, tankal naa la ndox

Pouvoir-tu-se.laver.exit-puiser.bén-1sg.perfect-toi-eau

Tu peux aller te doucher, j'ai puisé de l'eau pour toi

Pour ce qui concerne les phonèmes vocaliques, des changements sont aussi attestés. La voyelle centrale /ë/ et la postérieure /ó/ sont remplacées par la postérieure /u/. On remarque dans les exemples 21 et 23 que la mutation de /ë/ vers /u/ intervient dans un contexte particulier. En effet, on est en présence d'une conjugaison négative qui est peut-être à l'origine de ce changement. La

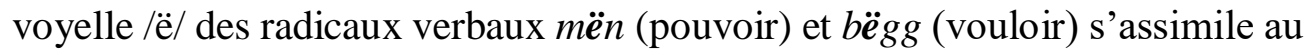
lieu d'articulation de la voyelle de la marque de négation '-u'. Pour le cas de l'exemple 23, il est vrai que nous ne sommes pas en présence d'une forme négative, mais la remarque reste la même. C'est dû à l'influence conditionnée par la négation qui a favorisé le changement de la voyelle /ó/ en /u/. Ce qui est important à noter à ce niveau c'est qu'elles sont toutes deux des voyelles postérieures.

21. Ñun munuñu lu dul kontaan si

Nous-pouvoir-1pl.conj.nég-ce-être.inacc-nég-content-y

Nous ne pouvons que nous en réjouir

22. La ти хат yёpp buggu ko wax

Ce que-il-savoir-tout-vouloir.nég-le-dire 
Il ne veut pas dire tout ce qu'il sait

\section{Jugal naan sa sirop}

Lever.imp-boire-ton-sirop

Lève-toi et prends ton sirop

Un autre changement observé est le cas de la voyelle antérieure /é/ qui devient une centrale /ë/. Beaucoup de mots du lexique wolof comportant cette voyelle subissent ce changement. Pour s'assurer qu'il ne s'agit pas de variantes régionales, on a eu recours au dictionnaire wolof - français de Arame Fal et Rosine Santos (1990) afin de vérifier nos doutes. C'est un changement qui s'est opéré et dont les locuteurs ne semblent pas conscients. Dans les exemples 24 à 28 on relève quelques mots qui, dans le dictionnaire ne présentent pas de variantes. Ce qu'on doit respectivement avoir c'est : fés (être très visible) ; ñépp (tout le monde) ; mébét (projet) ; fél (heurter) et wér (bonne santé). Pour certains mots, le fait de changer la voyelle /é/ en /ë/ change complètement leur sens. C'est le cas de wër (exemple 28) qui signifie « faire le tour de ».

24. Bii dafa fës jëlal bee

Celui-ci-il-être.très.visible-prendre.imp-celui-là

Celui-ci est trop visible prends celui-là

25. Li ñu bëgg mooy ñ̈̈pp jàpp si

Ce que-nous-vouloir-c'est-tous-prendre-y

Nous voulons le soutien de tout un chacun

26. Yaw kon sa mëb̈̈t mooy France rekk

Toi-donc-ton-projet-c'est-France-seulement

Donc ton objectif c'est uniquement aller en France

27. Moytul fël telefon bi

Éviter.imp-heurter-téléphone-le

Attention au téléphone

28. Yal na Yàlla guddal say fan te yokk la wër

Puisse-3sg.conj.perfec.-Dieu-allonger-tes-jours-et-ajouter-

bonne.santé

Puisse Dieu t'accorder longue vie et bonne santé

La réalisation [ё] en finale absolue n'est plus assurée pour certains mots comme sëdda [sëd:ë] (courbine) et gëdda [gëd:ë] (respect). En effet, chez certains locuteurs, on assiste à la chute de celle-ci en position finale. Exemple :

29. Kii de joxul benn nit gëdd

Lui-partic.disc.-donner.nég-aucune-personne-respect

Il ne respecte personne

Tel que noté dans 29, le mot gëdd signifie gronder et non pas respect. 
La voyelle centrale /a/ finale du morphème de l'interrogation ana (où) devient une antérieure /i/, comme le montrent l'exemple 30. C'est une façon de prononcer qui est très courante, particulièrement notée chez les jeunes filles qui veulent afficher une manière d'être raffinée.

30. Ani foto bébé bu ñaaw bi boog

Où-photo-bébé-qui-vilain-alors

Alors, où sont les photos du vilain bébé

\subsubsection{Les changements morphosyntaxiques}

Les changements observés dans cette catégorie concernent la variation lexicale en rapport avec l'environnement. La langue étant considérée comme un système de signes, il existe entre les éléments un ensemble de relation qui les lient les uns par rapport aux autres. En wolof, c'est le cas de la relation entre le nom et ses déterminants, le sujet et son verbe, etc. Cependant, l'on observe dans le «wolof urbain contemporain » des écarts de langage aussi bien dans la flexion nominale que verbale. Ainsi a-t-on respectivement relevé une tendance de réduction de l'ensemble des 8 classes nominales du singulier en une seule classe ; une généralisation de l'usage de l'épenthèse '-j', placé devant la marque '-i' que Diouf (2009, p. 56) appelle «exitif» ou « exportatif » pour les verbes se terminant par une voyelle ; l'absence d'accord entre le verbe et son sujet; l'abandon de l'usage du joncteur verbal 'a' ; l'amalgame qui est fait entre les pronoms personnels $1 \mathrm{pl}$ et $3 \mathrm{pl}$ et enfin la marque de l'impersonnel '-ees' 3sg combinée au pronom $3 \mathrm{pl}$.

Dans les exemples 31 à 34 , on a un ensemble de mots appartenant à des classes nominales différentes (at $\boldsymbol{m i}$ 《l'année », nit $\boldsymbol{k i}$ 《la personne », doom ji « l'enfant», fas wi « le cheval»), qui se retrouve partageant une même classe à savoir la classe « B- ». La cause probable est à chercher du côté de l'existence de mots d'emprunt qui sont tous rangés dans le tiroir de cette classe nominale. Il y a énormément de mots étrangers dans le lexique wolof et la fréquence d'usage de ces derniers peut être à l'origine de cette tendance qui s'est bien implantée maintenant.

31. At bu jot ñu màggal ko

Année-qui-arriver-nous-célébrer-le

On le célèbre chaque année

32. Nit bu dëddu àdduna ku ko miraasul du tàlli

Personne-qui-quitter-monde-celui-le-partager.biens-être.nég-

être.tranquil

Un défunt dont on n'a pas partagé l'héritage à ses enfants ne reposera pas en paix

33. Yaroo sa doom bi de

Eduquer.nég-ton-enfant-le-patic.disc.

Tu n'as vraiment pas éduqué ton enfant 


\section{Fas bii laa la doon wax moo am doole}

Cheval-ce-1sg.emph.comp.-te-être.pass-parler-il-avoir-force

C'est ce cheval que je te disais être fort

Parfois, pour certains mots, le fait de changer sa classe d'appartenance, change complètement sa signification. Cela fait partie d'ailleurs des procédés de formation de nouvelles unités lexicales en wolof et est connu sous le nom « dérivation impropre » ou «inversion»; c'est-à-dire par changement de classe. C'est le cas qui se présente en 33 : doom avec la classe 'bi' renvoie au fruit de l'arbre, mais avec 'ji' cela renvoie à la progéniture de l'être humain.

Dans l'exemple 35 on assiste à une situation particulière avec le pluriel de certains noms se rattachant à des humains à l'instar de nit (personne), góor (homme), jigéen (femme), gan (invité), etc. qui ont toujours ' $\tilde{N}$-' comme pluriel et non 'Y-' comme le montre l'exemple. La construction correcte est : Noo ngi dàq nit $\tilde{\boldsymbol{n} i}$.

35. Noo ngi dàkk nit yi

Ils-prés-chasser-personne-les

On chasse les gens

L'accord de classe, non plus, n'est pas toujours assuré. On observe des constructions du genre :

36. Seede yi benn la

Témoignage-les-un-être

Les témoignages sont les mêmes

37. Mooy tur wu bees bi nga xam ne...

C'est-prénom-qui-nouveau-le-tu-savoir-que

C'est le nouveau prénom dont...

38. La ти хат yёpp buggu ko wax

Ce.que-il-savoir-tout-vouloir.nég-le-dire

Il ne veut pas dire tout ce qu'il sait

En forme correcte les accords de classe donneraient : 36. Seede yi yenn lañu ; 37. Mooy tur wu bees wi nga xam ne ; 38. La mu xam lépp bëggu koo wax.

S'agissant de la consonne épenthétique '-j', placée entre la voyelle finale du radical verbal et le morphème '-i' de «l'exitif» qui renvoie à l'idée de " aller faire », on remarque qu'elle n'est plus réservée à ce genre de verbe. Son usage est devenu général, car étant utilisé même pour les verbes qui se terminent par une consonne ; ce qui ne devrait pas être le cas. Les verbes (màggal «célébrer », ndugg "faire les courses », jox «donner », sëy «se marier», jënd «acheter»), qu'on observe dans les exemples 39 à 42, ne devraient pas comporter cette forme en '-ji' dans la mesure où ils se terminent par une consonne. 
39. ...topp seen ginnaaw dem màggaliji

...suivre-leur-derrière-aller-célébrer

....les suivre pour aller au magal

40. Dinañu ko jënd bu ñuy nduggiji

1pl.fut-le-acheter-quand-nous-faire.courses

Nous l'achèterons lorsque nous irons faire les courses

41. Pàppaam ak yaayam dañoo divorcer, yaayam dem sëyiji Tuubaa Père.3sg.poss-et-mère.3sg.poss-ils-divorcer-mère.3sg.poss-allerse.marier-Touba

Son père et sa mère ont divorcé et sa mère est partie se marier à Touba

42. Dafa am benn mótóor bu may jëndiji

Il-avoir-un-moteur-que-moi.inacc-acheter

J'ai un moteur à aller acheter

Les formes correctes étant: màggali «aller célébrer», nduggi «aller faire les courses », joxi aller donner », sëyi «aller se marier », jëndi «aller acheter ».

L'accord au pluriel entre le verbe et son sujet a également tendance à diminuer. En effet, comme le montrent les illustrations 43 à 46, les locuteurs, dans la conjugaison, font maintenant usage de noms au pluriel sans les accorder avec leurs verbes. C'est un fait qui est devenu habituel chez beaucoup de locuteurs prouvant ainsi que certaines règles de grammaire de la langue ont tendances à être rangées aux oubliettes. La construction correcte pour chacun de ces exemples étant : Sama bët yi ndaw nañu... (mes yeux sont petits...); barabi fajukaay yi doyuñu... (les centres de santé sont insuffisants...) ; yitte yi duñu jeex (les besoins/préoccupations ne finissent jamais); kër yi ci boppam ñoo toj (les familles sont en désordre).

43. Sama bët yi ndaw na waaye man nañoo gis de

Mon-oeil-les-petits-3sg.conj.perfec-mais-pouvoir-3pl.conj.perfecvoir-partic.insist

Mes yeux sont petits mais ils voient bien

44. Barabu fajukaay yi doyul

Lieu.conn-soigner.instr-les-suffir.nég

Les centres de santé ne sont pas suffisants

45. Yitte yi du jeex

Besoins-les-être.nég-finir

Les besoins/préoccupations ne finissent jamais

46. Kër yi ci boppam moo toj

Maison-les-prép.loc-tête.3sg.poss-il-casser

Les familles sont en désordre 
Dans l'exemple 44, on constate que la marque du connectif, qui sert à construire le complément du nom, ne s'accorde pas au nom qu'il détermine, malgré le fait que celui-ci soit au pluriel. La construction correcte est : barabi fajukaay yi... (les centres de santé...).

L'absence d'accord au pluriel est aussi observée avec le pronom objet. Dans les exemples 47 à 49, les noms auxquels se rapportent les pronoms objets sont au pluriel donc ceux-ci devraient s'accorder en nombre. Ce qui donnerait le pronom leen pour le pluriel à la place de ko réservé au singulier.

47. Pàkkam yi mu jot a am yëpp jaay na ko

Parcelles-les-il-recevoir.déjà-jonc-avoir-tout-vendre-3sg.conj.perfecle

Il a vendu toutes les parcelles qu'il avait 48. Ay am-amam, ay jabaram, ay otoom, lijjantiwuma ko

Art.ind.pl-richesse.3sg.poss-art.ind.pl-épouse.3sg.poss-art.ind.plvoiture.3sg.poss-s'occuper.1sg.conj.nég-le

Je ne m'occupe pas de ses affaires

49. Dàll yi maa ko moom

Chaussure-les-moi-le-posséder

Les chaussures sont à moi

Le joncteur verbal 'a' qui, selon le contexte, se présente sous la forme 'a' lorsqu'on est en présence de deux verbes distincts ou '-oo' lorsqu'il s'agit du pronom objet $k o$, a lui aussi tendance à être omis par certains locuteurs. Les exemples 50 et 51 présentent tous deux une incorrection avec l'absence de ce monème fonctionnel. Cependant, le premier cas, c'est-à-dire l'exemple 50, présente plus d'ambiguïté parce que le sens véhiculé dans ce contexte n'est pas rendu dans la traduction. En effet, cette construction Dama bëgg dem Dakaar véhicule le sens de « j'aime aller à Dakar » qui est différent de Dama bëgg a dem Dakaar « je veux aller à Dakar».

Pour l'exemple 51, il s'agit d'une situation où les deux verbes sont séparés par un pronom objet. Dans ce cas précis, c'est ce dernier qui doit porter le monème fonctionnel ' $a$ ', raison pour laquelle nous devrions retrouver une longueur vocalique au niveau du pronom : Munuma koo gis... (je n'arrive pas à le voir...).

50. Dama bëgg dem Dakaar suba

Je-vouloir-partir-Dakar-demain

Je veux partir à Dakar demain

51. Munuma ko gis si mbedd bi

Pouvoir.1sg.conj.nég-le-voir-dans-rue-la

Je n'arrive pas à le voir dans la rue 
La forme yeena de la conjugaison emphatique du sujet $3 \mathrm{pl}$ a subi un changement particulier très courant, surtout chez les jeunes. La forme yaa leen par laquelle elle est substituée correspond respectivement à l'emphatique du sujet 2 sg et au pronom objet 2 pl ou 3 pl. On est en présence d'un exemple typique d'incorrection qui ne véhicule aucun sens et qui mérite d'être signalé.

52. Yeen ñi nga xam ne yaa leen si jàppaale

Vous-qui-tu-savoir-que-2sg.emph.suj-leur-y-soutenir.conco

Vous qui nous avez soutenus

La forme '-ees' de la marque de l'impersonnel qui doit toujours être accompagnée du pronom 'na' de la conjugaison perfective se retrouve de manière très courante combinée au pronom 'nañu' 3 pl comme l'illustrent les énoncés 53 et 54. Cela indique une méconnaissance des règles grammaticales par certains locuteurs. En effet, cette marque '-ees' est un morphème de troisième personne du singulier et correspond au pronom impersonnel « on » du français. Il doit toujours être suivi de 'na' (conjugaison perfective $3 \mathrm{sg}$ ).

53. Manees nañoo wax ni...

Pouvoir.3sg.impers-nous-dire-que

On peut dire que...

54. Ci sama xalaat, warees nañoo bàyyi ndaw yi ci seen sago

Prep.loc-mon-avis-devoir.3sg.impers-nous-laisser-jeunes-lesprép.loc-leur-liberté

A mon avis on doit laisser aux jeunes leur liberté

Nous terminons cette liste par l'amalgame qui est fait entre les pronoms sujets $1 \mathrm{pl}$ et $3 \mathrm{pl}$. Beaucoup de locuteurs ne font plus la distinction entre ces deux pronoms. En effet, la majeure partie des locuteurs emploie le pronom $\tilde{n} u$ indifféremment du contexte. Qu'il s'agisse de la première ou de la troisième personne du pluriel, c'est la même forme qui est utilisée. Ce faisant, il n'y a que le contexte qui puisse aider à déterminer s'il s'agit de « nous » ou « ils ». Cependant, il importe de souligner que cette différence était bien marquée jadis avec $n u$ pour indiquer la première personne du pluriel et $\tilde{n} u$ la troisième.

\section{Am nañu droit wax ne ñun ànduñu ci}

Avoir-nous-droit-dire-que-nous-être.d'accord.nég-y

Nous avons le droit de dire que nous ne sommes pas d'accord La construction correcte serait : am nanu droit wax ne nun àndunu ci. L'ensemble de ces faits attestés, partant des phénomènes sociolinguistiques en passant par ceux purement linguistiques, ont des causes mais aussi des conséquences sur la langue et sa communauté. 


\section{Les causes et conséquences de ces phénomènes}

\subsection{Les causes}

Elles sont multiples. L'on peut citer, dans cette liste non exhaustive, les mouvements de populations (mondialisation), le contact de langues, le complexe d'infériorité, l'appartenance linguistique de l'interlocuteur, l'existence de nouvelles réalités que la langue doit nommer, le développement des cultures urbaines, le problème de la transmission, le rôle des politiques linguistiques.

Les Wolof constituent un groupe qui a une longue culture migratoire. Ils se déplacent beaucoup vers des endroits propices à leurs activités économiques aussi bien au Sénégal qu'à l'étranger. En bons commerçants, ils ont toujours sillonné l'intérieur du pays et ont été ainsi en contact avec d'autres communautés locales. Ils sont également de grands voyageurs vers les pays développés. Au Sénégal comme à l'étranger, la rencontre avec d'autres communautés favorise inévitablement le contact de langues, phénomène qui a toujours existé partout à travers le monde. Les locuteurs wolof immigrés et leurs enfants ont une façon de parler dans laquelle on note un certain degré d'interférence. Les enfants nés en France ont un discours particulier ; comme celui de ce petit garçon rencontré lors d'un voyage d'étude qui s'adressait à sa mère :

56. J'ai mouillé mon pantalon en me wisuwant (wisu : s'humecter après avoir fait pipi)

57. C'est elle qui me xosi (xosi : griffer)

Nous assistons dans l'exemple 56 à un cas d'hybridation lexicale avec le mot wisuwant, mais dans cette combinaison d'éléments, nous avons un radical verbal provenant du wolof (wisu: s'humecter) suivi du participe présent (-ant) provenant du français.

Le brassage entre communautés à travers les mariages mixtes est aussi un fait non négligeable qui favorise le contact de langues et cela figure parmi les causes des changements qui s'opèrent dans le «wolof urbain contemporain ». Le fait de s'unir à des personnes de langue maternelle différente conduit le locuteur wolof à perdre plus ou moins les normes d'usage de sa langue.

En outre, il y a le complexe d'infériorité qui explique en partie le nonrespect des règles grammaticales des locuteurs wolof. Beaucoup d'entre eux, pour éviter de se faire passer pour des campagnards, préfère s'exprimer en ayant recours à «l'emprunt de luxe » aux langues étrangères. Ils n'hésitent pas non plus à déformer la prononciation des vocables wolof ou bien même l'intonation de leur voix juste pour s'identifier à des gens « civilisés », venant de la «haute société ». Ce complexe d'infériorité est plus visible lorsque le locuteur wolof est en face d'un interlocuteur de langue différente. Il s'adapte 
généralement à la façon dont l'interlocuteur s'exprime au détriment des règles grammaticales que lui impose sa langue.

L'existence de nouvelles réalités que la langue doit nommer fait également partie des causes. Avec l'évolution du monde, de nouvelles réalités voient le jour. Les locuteurs n'étant pas en mesure de les nommer dans leur propre langue n'ont aucune autre alternative que de les utiliser tel qu'elles leur arrivent. C'est aussi le cas pour ce qui concerne le développement des cultures urbaines (dans les domaines de la mode, de la musique, de l'artisanat, des nouvelles technologies de l'information et de la communication, etc.). Les locuteurs ne font pas l'effort de chercher s'il existe, dans le répertoire de leur langue, des termes qui correspondraient au lexique provenant de ces nouvelles cultures étrangères.

Le problème de la transmission est aussi un facteur notoire. En effet, il faut chercher à savoir quelle façon de parler (quel wolof ?) a été transmise à ces jeunes générations de locuteurs. S'il s'avère qu'il s'agit du wolof hybride avec les interférences et les écarts grammaticaux, l'on ne pourrait s'attendre à ce que ces générations s'expriment dans un wolof « authentique ».

L'absence de politiques linguistiques pertinentes et durables peut également être lister parmi les causes. Si l'État ne pose pas des actes forts, notamment l'officialisation des langues nationales et leur introduction effective dans l'éducation, allant dans le sens d'œuvrer pour leur promotion, cette situation ne peut que perdurer.

Tous ces faits auront bien évidemment des conséquences sur la langue et sa communauté.

\subsection{Les conséquences}

Il est clair que les langues naissent naturellement, évoluent et parfois même certaines meurent et que partout où il $\mathrm{y}$ a contact entre groupes sociaux il y a inévitablement influence sur le plan linguistique et culturel. L'évolution d'une langue qui tire son origine de son contact avec d'autres langues a forcément des conséquences sur l'avenir de celle-ci et de sa communauté. Ainsi, il importe d'alerter l'opinion sur ces conséquences. C'est d'ailleurs la position de Fallou Ngom (2005, p. 163) qui soulève dans son étude des questions très pertinentes méritant d'avoir l'attention de tous les chercheurs intéressés par ce phénomène. En se demandant : le « wolof va-t-il disparaître en tant que langue (lexicalement et grammaticalement) distincte du français, ou va-t-il devenir une langue sous-régionale fortement 'colorée' par le français, l'anglais et l'arabe ? ", il tire en quelque sorte sur la sonnette d'alarme. Cela veut dire que, suivant le degré d'évolution de ces changements, on risque d'assister à l'extinction du « vrai » wolof ou bien de se retrouver tout simplement avec une langue qui aurait une appellation autre que 'wolof'. Cette «langue colorée» dont parle Ngom n'est que la partie visible de 
l'iceberg. Nous avons, dans la partie cachée, la non maîtrise et/ou l'oubli du vocabulaire et des règles de grammaires qui va crescendo. Les locuteurs wolof sont influencés par les langues qui les entourent et ce contact permanent conduit généralement à une perte considérable du stock de leur vocabulaire, voire la non maîtrise des normes d'usage de la langue. Si on ignore le vocabulaire et certaines règles de grammaire de sa langue, on est obligé d'emprunter aux autres langues pour pouvoir communiquer et cela mène inéluctablement à commettre des incorrections et ainsi «massacrer »sa langue.

Cependant, l'espoir est toujours permis et il incombe à tous les acteurs de faire des efforts pour remédier à cette situation qui peut conduire à une « insécurité linguistique».

\section{Les perspectives}

A la lumière des descriptions faites, le wolof urbain contemporain, qui fait l'objet d'une pratique courante partout à travers le Sénégal, est marqué par des interférences et des incorrections. A notre avis, la première attitude à avoir face à cette situation c'est d'alerter la communauté qui semble bien inconsciente des «dérives» commises. Il incombe à tous les acteurs, les composantes de la communauté wolof, les chercheurs, de même que les autorités en charge des politiques linguistiques, de collaborer afin de se donner les moyens d'alerter, d'encadrer et de veiller à l'application des bonnes pratiques linguistiques. Ceci est également valable pour les autres langues nationales qui sont dans la même position que le Wolof. Il incombe à chaque locuteur de tout faire pour protéger et promouvoir sa langue. L'alerte consiste également à inciter les locuteurs à dénoncer la négligence par rapport au respect des règles ; à consulter des personnes ressources qui parlent bien la langue et à avoir plus de considération pour elle en se gardant de faire des fautes et des interférences exagérées, comme ils se garderaient d'en faire s'il s'agissait d'une langue étrangère.

Il urge également de repenser les politiques linguistiques concernant l'introduction des langues nationales dans le système éducatif en tenant compte, bien évidemment, de l'environnement sociolinguistique.

D'après certains théoriciens de l'acquisition du langage, notamment les innéistes, la langue s'acquière de manière naturelle, mais pour d'autres comme les interactionnistes l'environnement y joue un rôle déterminant. En se fondant sur cette dernière perception, on peut affirmer qu'il est possible de remédier à cette situation «d'insécurité linguistique » que connait la langue wolof en rendant effectif et immédiat l'enseignement des langues nationales dans tous les niveaux du système éducatif sénégalais comme médium et objet d'enseignement. Ce serait ainsi l'occasion pour les locuteurs de découvrir les normes qui régissent le fonctionnement de leurs langues. Cependant, cela 
nécessite une réelle organisation et de la volonté politique. Il faudrait, en effet, des mesures d'accompagnement telles que l'élaboration de programmes d'enseignement, d'un cadre de référence pour l'enseignement et l'apprentissage des langues nationales et des ressources pédagogiques pertinents pour qu'une telle entreprise soit une réussite.

\section{Conclusion}

Dans cet article on s'est intéressé à deux aspects de l'usage du wolof en milieu urbain : les changements d'ordre sociolinguistique et ceux d'ordre essentiellement linguistique. Ces changements sont issus d'un contexte de contact de langues où le wolof est en éternelle cohabitation avec des langues locales et étrangères, ce qui n'est pas sans conséquence. En effet, il ressort de l'analyse des données qui ont servi de corpus que la langue wolof présente un réel dynamisme. On a remarqué qu'il existe une grande différence entre le wolof « authentique » et celui parlé en zones urbaines de nos jours. Ce dernier s'éloigne de plus en plus des normes d'usage de la langue. La description de l'ensemble des faits attestés: emprunts, code-switching, code-mixing, changements phonologiques et morphosyntaxiques témoigne de ce dynamisme qui, semble-t-il, incite à croire qu'un tel usage risque de conduire à une "insécurité linguistique » vu la vitesse de l'évolution. Il est vrai que le contact de langues est inévitable, il est également vrai que les langues évoluent, mais de quel type d'évolution s'agit-il ? Faudrait-il laisser la langue évoluer vers de nouvelles « fausses » règles au détriment des normes d'usage ?

\section{References:}

1. Blanchet, P. (2012). La linguistique de terrain, méthode et théorie : une approche ethno-sociolinguistique de la complexité. $2^{\text {ème }}$ édition. Rennes : Presses universitaires de Rennes.

2. Calvet, L. J. (2004). La diversité linguistique : enjeux pour la Francophonie. Hermès, La Revue, vol. 40, n 3, 287-293.

3. Daff, M. (1998). L'aménagement linguistique et didactique de la coexistence du français et des langues nationales au Sénégal. DiversCité Langues, vol. 3, http://www.uquebec.ca/diverscite

4. Diagne, P. (1971). Grammaire de wolof modern. Paris : Présence africaine.

5. Diouf, J. L. (2009). Grammaire du wolof contemporain. Paris : L'Harmattan.

6. DGLFLF. (2005). Guide des bonnes pratiques pour la constitution, l'exploitation, la conservation et la diffusion des corpus oraux. Paris : CNRS.

7. Dumont, P. (1983). Le français et les langues Africaines au Sénégal, Paris : Karthala- ACCT. 
8. Fal, A., Santos, R. \& Doneux, J. L. (1990). Dictionnaire woloffrançais suivi d'un index français-wolof. Paris : Karthala.

9. Kébé, A. B. (2011). Contacts de langues et médias : le discours journalistique en wolof à l'épreuve du parler ordinaire sénégalais. Glottopol, $\mathrm{n}^{\circ} 18,21-33$.

10. McLaughlin, F. (2008). On the origins of urban Wolof: Evidence from Louis Descemet's 1864 phrase book. Language in Society n ${ }^{\circ} 37,713-$ 735 .

11. Ngom, F. (2005). Les Implications sociolinguistiques des emprunts du wolof au français. Revue internationale de langues, littératures et cultures, $\mathrm{n}^{\circ} 4,147-164$.

12. Thiam, N. (1990). L'évolution du wolof en milieu urbain sénégalais : le contexte dakarois. Plurilinguismes, $\mathrm{n}^{\circ} 2,10-37$.

13. Thiam, N. (1994). La variation sociolinguistique du code mixte woloffrançais à Dakar : une première approche. Langage et société, $\mathrm{n}^{\circ} 68$, $11-34$. 